\title{
Estrategias para Incentivar la Participación de Alumnos en Educación a Distancia
}

\author{
Marcus de Melo Braga ${ }^{1}$, Tatiana Takimoto ${ }^{2}$, Daniell Pontes Silva ${ }^{1}$, \\ Olival de Gusmão Freitas Junior ${ }^{1}$, Petrucio Antonio Medeiros Barros ${ }^{1}$ \\ ${ }^{1}$ Instituto de Computação - Universidade Federal de Alagoas (UFAL) \\ Maceió - Alagoas - Brazil \\ ${ }^{2}$ Instituto Euvaldo Lodi (IEL) \\ Florianópolis - Santa Catarina - Brazil \\ marcus@ic.ufal.br, tatiana.takimoto@ielsc.org.br, \\ daniell.pontes@sinfra.ufal.br, \{olival, petrucio.barros\}@ic.ufal.br
}

\begin{abstract}
Distance Education in Brazil has grown significantly over the last years. Just as in traditional courses, the activities of Distance Education also have student's dropout. This paper presents a proposal for the application of motivational strategies adopted to encourage participation of members of Communities of Practice to the activities of Distance Education as an approach to promote participation and reduce the dropout of students in distance courses. Taking as a starting point a literature review of the study of the barriers and the motivating factors in virtual communities, it is proposed and justified the adoption of a strategy based on these factors, to minimize the causes of student's dropout in Distance Education.
\end{abstract}

Resumen. En Brasil la Educación a Distancia ha crecido significativamente durante los últimos años. Así como en los cursos tradicionales, en las actividades de Educación a Distancia también hay deserción de alumnos. Este trabajo presenta una propuesta de aplicación de estrategias de motivación, adoptadas para incentivar la participación de miembros en Comunidades de Práctica, a las actividades de Educación a Distancia como una forma de favorecer la participación y reducir la deserción de alumnos en cursos a distancia. Tomando como punto de partida la revisión de literatura sobre el estudio de barreras y factores de motivación en comunidades virtuales, se propone y justifica la adopción de una estrategia basada en esos factores para minimizar las causas de la deserción de alumnos en Educación a Distancia.

\section{Introducción}

Los cursos de Educación a Distancia han crecido considerablemente en el país en los últimos años. Según el INEP (2013), el numero de alumnos que cursan la modalidad de Educación a Distancia corresponde ya a más del 15\% del volumen total de las carreras de grado en el país. Sin embargo, aunque haya crecido bastante la oferta de enseñanza superior por Educación a Distancia, la modalidad aún enfrenta algunos problemas de gestión, como el de la deserción. 
La deserción en la Educación a Distancia $(\mathrm{EaD})$ es un tema relevante, ya que implica un bajo desempeño institucional, producido por la disminución del número de alumnos matriculados y el desperdicio de recursos financieros aplicados a la educación superior. Algunos autores consideran que las deserciones son elevadas [Favero y Franco, 2006; Levy, 2007; Santos y Oliveira Neto, 2009]. Independientemente del orden de su magnitud, empero, la deserción es un efecto que debe ser combatido, una vez que representa un desperdicio de recursos. En ese sentido, para el estudio del tema es importante cualquier iniciativa que busque aumentar la efectividad de los procesos de Educación a Distancia posibilitando la reducción de la deserción.

En el presente artículo se toman como punto de partida los estudios de las estrategias de motivación adoptadas en las Comunidades de Práctica, proponiendo que sean adoptadas en las actividades de Educación a Distancia a fin de reducir la deserción en esos programas.

\section{Trabajos Relacionados}

Varios estudios recientes se han abocado a esa cuestión, principalmente en la EaD: Detoni et al. (2014), Heidrich et al. (2014), Manhães et al. (2011) y Ramos et al. (2014).

Al analizar la deserción, Ramos et al. (2014) identifican algunas barreras en la $\mathrm{EaD}$, pero se concentran principalmente en las dificultades de desempeño de los alumnos en la Educación a Distancia. Detoni et al. (2014) estudian la predicción de reprobación de alumnos en la $\mathrm{EaD}$ con base en las técnicas de aprendizaje de máquina, usando el conteo de las interacciones de los alumnos en el Ambiente Virtual de Aprendizaje (AVA). Heidrich et al. (2014) estudian los efectos de los Estilos de Aprendizaje en la deserción de alumnos en la $\mathrm{EaD}$ y proponen un modelo que recoge datos del AVA y usa Rutas de Aprendizaje y Estilos de Aprendizaje para hacer inferencias sobre el desempeño de los alumnos. Finalmente, Manhães et al. (2011) usan la técnica de minería de datos para prever el riesgo de deserción de alumnos en la EaD.

\section{Educación a Distancia}

Según Peters y Keegan (1994), la EaD es una forma de educación en la cual estudiantes de universidades no aprenden en salones de clase sino que reciben material didáctico elaborado por profesores y asistentes con conocimientos de Educación a Distancia. Sin embargo, el concepto de mayor alcance es dado en Moore (2007): el estudiante se encuentra en un sitio distinto del profesor y el uso de medios electrónicos para el seguimiento del aprendizaje no es una alternativa sino una característica determinante de la relación entre alumnos y profesores.

Para Almeida (2003), la falta de interacción entre las personas es uno de los factores de desmotivación en las actividades de EaD y ocasiona altos índices de deserción y poca productividad. Moran (2007), por su parte, al exponer los modelos de $\mathrm{EaD}$, sugiere que el éxito de los cursos en línea depende de la integración del grupo y su colaboración. De acuerdo con el autor, el énfasis debe ser dado más en la colaboración que en la lectura de textos. Las discusiones y actividades en colaboración terminan 
construyendo parte del curso, haciéndolo más atractivo para el mismo grupo. En ese contexto las comunidades virtuales creadas durante el curso pueden ser vistas como "comunidades de práctica de aprendizaje". Según Palloff y Pratt (2007), una comunidad de aprendizaje en línea es un espacio donde alumnos y docentes conversan e intercambian informaciones como iguales, al tiempo que se perciben trabajando juntos con el mismo objetivo. Para Wenger (2010), los conceptos de interacción, colaboración, participación, cooperación e interés común en el aprendizaje son inherentes al término Comunidad de Práctica.

\section{Comunidades de Práctica}

Comunidades de Práctica $(\mathrm{CoP})$ son redes informales de colaboración que ofrecen apoyo a los participantes de una profesión, en sus esfuerzos por desarrollar comprensión en común y en su comprometimiento con la construcción de un conocimiento relevante para sus trabajos, según Hara (2009).

Las actividades de Educación a Distancia pueden propiciar la formación de comunidades de práctica entre los alumnos, aunque sean temporales. En las CoP, los novatos comparten el conocimiento de los miembros con más experiencia, por medio de discusiones en línea y por el intercambio de mensajes entre los varios integrantes. En esas interacciones, el conocimiento es transmitido y compartido, posibilitando el aprendizaje.

\section{Motivación en las Comunidades de Práctica}

Para estimular la participación en una comunidad, se puede adoptar una serie de actividades bastante similar a las que se adoptan en las comunidades presenciales. Con base en los estudios de McKenzie-Mohr y Smith (1999), es posible establecer cinco etapas en este proceso de motivación:

1) Identificar barreras para la participación;

2) Identificar incentivos para la participación;

3) Desarrollar una estrategia de participación considerando:

a. El comportamiento social/cultural del grupo;

b. Las dificultades/barreras para la participación;

c. Los factores facilitadores/motivadores de la participación;

d. La participación de minorías;

e. La creación de incentivos/recompensas;

f. El reconocimiento público por la participación;

4) Conducir la estrategia de participación;

5) Evaluar la estrategia de participación.

\subsection{Barreras para la Participación}

En la literatura sobre $\mathrm{CoP}$ existen varios estudios que abordan las principales barreras que deben trasponerse para su correcto cultivo, es decir, para que realmente sean efectivas [Wenger, McDermott y Snyder, 2002; Kimble, Li y Barlow, 2000; Wasko y Faraj, 2000; Goman, 2002; Ardichvili, Page y Wentling, 2003; Nonnecke et al 2004; Gannon-Leary y Fontainha, 2007]. 
Ardichvili (2008) propone una clasificación, en cuatro grandes grupos, de las barreras para la participación en Comunidades Virtuales: interpersonales, procesales, tecnológicas y culturales. Consideramos como barreras interpersonales aquellas relacionadas con las dificultades de participación propias del individuo o de su interrelación con los demás. Las barreras procesales son las ocasionadas por los procesos y estructura organizacional y sus respectivos controles. Las barreras tecnológicas están asociadas a las dificultades en el uso de los recursos tecnológicos, específicamente los de la plataforma de software o de los recursos de la red de telecomunicaciones. Por fin, las barreras culturales están ligadas a cuestiones relacionadas con las diferencias culturales y sociales entre los participantes.

Las Tablas 1, 2, 3 y 4 presentan las barreras interpersonales, culturales, tecnológicas y procesales en una comunidad virtual, agrupadas de acuerdo con la nomenclatura propuesta en Ardichvili (2008).

TABLA 2. Barreras Culturales

TABLA 1. Barreras Interpersonales

\begin{tabular}{l}
\hline Barreras Interpersonales \\
\hline 01. Falta de confianza en el propio \\
conocimiento \\
02. Nada que ofrecer \\
03. No estar seguro de la exactitud \\
04. Miedo de "errar el tiro" \\
05. Miedo de dar una información equivocada \\
06. Temor de que la contribución no sea \\
importante o apropiada \\
\hline 07. No verse aún en el derecho a contribuir \\
\hline 08. Estar aun aprendiendo con el grupo \\
\hline 09. Deseo de continuar siendo anónimo \\
\hline 10. Vergüenza de contribuir \\
\hline 11. Temor sobre el uso que se dará a la \\
contribución \\
\hline 12. No hay pedidos de contribuciones \\
\hline 13. Olvido de contribuir \\
\hline 14. El trabajo extra y la responsabilidad \\
resultantes de la contribución \\
\hline 15. Ausencia de encuentros "cara a cara" para \\
romper el hielo \\
\hline 16. Falta de confianza en las personas \\
17. Falta de compromiso con el grupo \\
18. Las personas trabajan para otros que no \\
\hline comparten sus conocimientos \\
\hline
\end{tabular}

\section{Barreras Culturales}

01. Preguntar asuntos que están en los manuales

\begin{tabular}{l}
\hline 02. Ayudar sólo a quien nos ha ayudado antes \\
\hline $\begin{array}{l}\text { 03. Postura arrogante de los miembros } \\
\text { experimentados }\end{array}$ \\
\hline 04. Otros lo dirán \\
\hline 05. El grupo trata mal a los novatos \\
06. No tener la intención de publicar \\
\hline 07. No gano nada con eso \\
08. Los mensajes son de baja calidad \\
09. Estar en el grupo equivocado \\
\hline 10. Con solo leer/navegar basta \\
\hline 11. Creencia de que el conocimiento es poder \\
sobre los otros \\
\hline 12. La alta especialización exigida \\
13. La cultura académica de independencia \\
\hline 14. Cambio de asociación de comunidades \\
\hline 15. Ocultar las informaciones como ventaja \\
competitiva
\end{tabular}

TABLA 3. Barreras Tecnológicas Barreras Tecnológicas

01. Mala infraestructura de red

02. Demora en las respuestas 
TABLA 4. Barreras Procesales

\begin{tabular}{|c|c|}
\hline \multicolumn{2}{|c|}{ Barreras Procesales } \\
\hline $\begin{array}{l}\text { 01. Gran volumen de mensajes en grupos } \\
\text { numerosos }\end{array}$ & 06. No hay tiempo para contribuir \\
\hline $\begin{array}{l}\text { 02. Mi empresa no me permite publicar } \\
\text { contribuciones }\end{array}$ & 07. Mucha dependencia de la coordinación \\
\hline $\begin{array}{l}\text { 03. Falta de claridad sobre qué es una } \\
\text { contribución aceptable }\end{array}$ & 08. Estratificación de la comunidad \\
\hline 04. No saber cómo contribuir con ese grupo & 09. Tentativa de administrar la comunidad \\
\hline $\begin{array}{l}\text { 05. Contribuciones pueden ser rechazadas por la } \\
\text { coordinación }\end{array}$ & $\begin{array}{l}\text { 10. Miedo de consecuencias negativas en la } \\
\text { empresa }\end{array}$ \\
\hline
\end{tabular}

La identificación de las principales barreras es relevante ya que tienen que ser superadas para que se incentive la participación y el libre flujo de ideas en la comunidad.

\subsection{Incentivos a la Participación}

Varios estudios abordaron los principales factores de incentivo a la participación o a la contribución de los miembros de una comunidad de práctica [Wenger, McDermott y Snider, 2002; Ardichvili, Page y Wentling, 2003; Gannon-Leary y Fontainha, 2007; Hoadley y Pea, 2001; Chan et al 2004; Teigland y Wasko, 2004; Beenen et al 2004; Mahar, 2007].

La Tabla 5 presenta un resumen de los principales factores de incentivo a la participación en las comunidades de práctica.

TABLA 5. Factores de incentivo a la participación en una CoP

\begin{tabular}{|c|c|}
\hline \multicolumn{2}{|c|}{ Incentivos a la Participación } \\
\hline 01. Contribución al bien colectivo & 16. Sentido de objetivo compartido \\
\hline 02. Creer que la contribución es única & 17. Forma parte del trabajo \\
\hline 03. Obtener conocimiento en general & 18. Mejora el desempeño profesional \\
\hline 04. Obtener respuestas para problemas de trabajo & 19. Crea sinergia \\
\hline 05. Construir relaciones profesionales & 20. Sentimiento de pertenecer al grupo \\
\hline 06. Ofrecer experiencia al grupo & 21. Aumento de insights entre los miembros \\
\hline 07. Mantenerse actualizado & $\begin{array}{l}\text { 22. Concepción del conocimiento como bien } \\
\text { público }\end{array}$ \\
\hline 08. Aprender a participar & 23. La empresa incentiva el apoyo mutuo \\
\hline 09. Sentido de comunidad/reciprocidad & $\begin{array}{l}\text { 24. Necesidad de ser reconocido como } \\
\text { especialista (reputación) }\end{array}$ \\
\hline 10. Sentido de la obligación & $\begin{array}{l}\text { 25. Tiempo de retribuir a los otros, compartiendo } \\
\text { el propio conocimiento }\end{array}$ \\
\hline 11. Identidad/identificación con el grupo & $\begin{array}{l}\text { 26. El sitio de la comunidad (sistema) es visto } \\
\text { como una enciclopedia }\end{array}$ \\
\hline 12. Excelencia dentro el grupo & $\begin{array}{l}\text { 27. El sistema es visto como una herramienta de } \\
\text { solución de problemas }\end{array}$ \\
\hline 13. Reconocimiento tangible & $\begin{array}{l}\text { 28. El sistema es visto como una herramienta de } \\
\text { actualización profesional }\end{array}$ \\
\hline 14. Aumento de la autoeficacia & $\begin{array}{l}\text { 29. El sistema provee un espacio para la } \\
\text { generación del conocimiento }\end{array}$ \\
\hline 15. Aumento de la autoestima & $\begin{array}{l}\text { 30. El sistema colabora con las necesidades } \\
\text { individuales de información }\end{array}$ \\
\hline
\end{tabular}


La relevancia de los factores de incentivo a la participación o contribución de los miembros de una CoP guarda relación, precisamente, con su directa aplicación a los objetivos de este artículo y, por consiguiente, a la propuesta de que sean adoptados en las actividades de Educación a Distancia para mejorar su efectividad y contribuir a la reducción de la deserción.

\section{Modelo Propuesto}

El modelo presentado es una adaptación de la propuesta presentada en Chan et al (2004), con inserción del tratamiento de las barreras para la participación ya identificadas en el presente estudio. La necesidad del tratamiento de los factores que no se consideran barreras se justifica por los mismos motivos que se consideran en el caso de las CoP: aumentar la motivación y, por consiguiente, la participación de los integrantes.

El modelo propuesto (Figura 1) sigue las cinco etapas ya presentadas al principio de la Sección 4. En él, empezamos con la etapa 1: identificación de las barreras. Esa etapa es esencial para la planificación de acciones que puedan eliminarlas o minimizarlas, reduciendo las dificultades de participación. Después del tratamiento de las barreras, se debe enfatizar la identificación de los factores que sirven de incentivo a la participación de las personas en las comunidades. Una vez identificadas las barreras y los incentivos a la participación, se desarrolla la estrategia para su promoción, tomando en cuenta los seis aspectos listados en la etapa 3. El paso siguiente consiste en la ejecución, es decir, en seguir la estrategia de participación y, por fin, tras su realización, se evalúa la estrategia de participación adoptada para tener insights y conocimiento sobre todo el proceso.

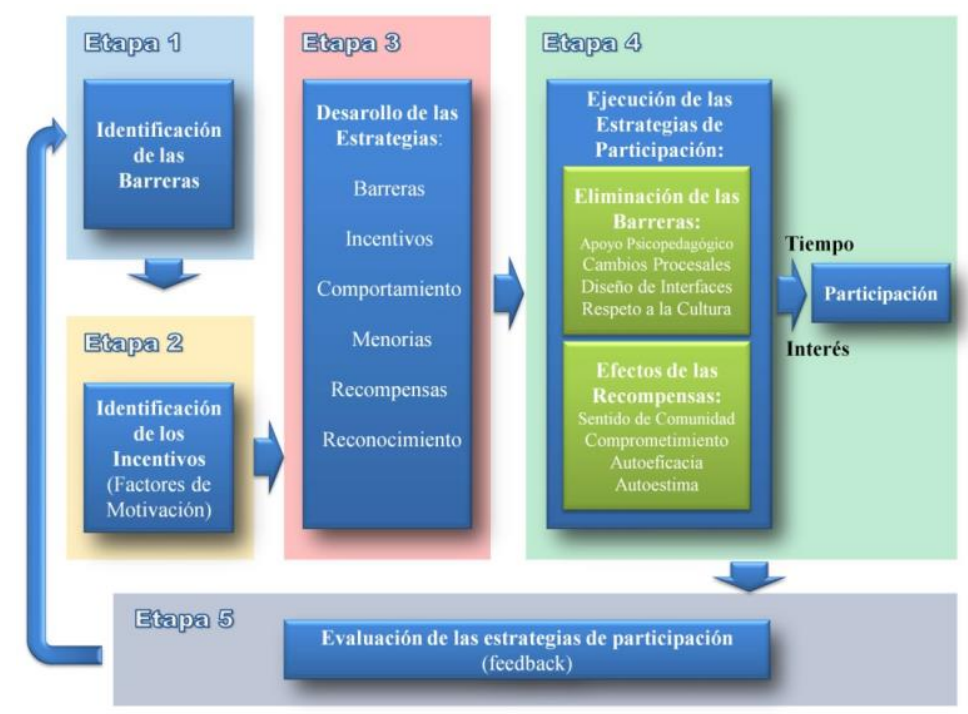

Figura 1. Modelo para incentive a la participación en EaD (adaptado de Chan et al, 2004)

\section{Etapa 1. Identificación y Eliminación de las Barreras}

La eliminación de las barreras es uno de los factores de gran importancia para el aumento del incentivo a la participación. Para cada clase de barrera se deben prever 
medidas rectificadoras que combatan o por lo menos mitiguen cada una de ellas. Las barreras interpersonales pueden ser combatidas con apoyo psicopedagógico; las procesales con cambios procesales que sean visibles; las barreras tecnológicas pueden ser minimizadas con el diseño de interfaces y capacitación y, por fin, las barreras culturales pueden ser sorteadas con el respeto a las diferentes culturas existentes en la comunidad.

\section{Etapa 2. Identificación y Adopción de los Incentivos/Recompensas}

En Chan et al (2004) se identifican las tres principales formas de recompensas o reconocimiento: identidad, excelencia y recompensas tangibles. La identidad se relaciona con la construcción de la identificación de cada miembro de la comunidad. Se opone al tradicional anonimato que algunas comunidades virtuales aún adoptan. La identificación de cada miembro contribuye a aumentar el interés en participar y contribuir con el grupo. La excelencia o la alta competencia de un experto (especialista) se alcanza a través del intercambio de conocimientos entre veteranos y novatos en una comunidad de especialistas. Por otra parte, las recompensas tangibles y el reconocimiento son poderosos instrumentos de incentivo a la participación.

Para Chan et al (2004) los efectos de las recompensas (o reconocimiento) son: (i) la creación de un "sentido de comunidad"; (ii) el comprometimiento; (iii) la mejoría de la autoestima y (iv) la de la autoeficacia. La autoeficacia está directamente asociada a la competencia o confianza en las capacidades personales y puede ser definida como el sentimiento de sentirse capaz de realizar una tarea o actividad con éxito. Autoeficacia es algo distinto de autoestima. La autoestima está asociada con la valoración personal mientras que la autoeficacia se relaciona con la habilidad de alcanzar metas.

\section{Etapa 3. Desarrollo de la Estrategia de Participación}

En esa etapa, todas las barreras e incentivos ya identificados en las etapas anteriores son tomados en consideración para el desarrollo de la estrategia de participación de los alumnos. Los estudios de McKenzie-Mohr y Smith (1999) prevén que en esta etapa se tome en cuenta el comportamiento social/cultural del grupo, la participación/inclusión de menorías y el reconocimiento, además de las barreras y factores de incentivo a la participación.

\section{Etapa 4. Ejecución y Evaluación (5)}

Tras el estudio cuidadoso de la estrategia de participación, se pasa finalmente a la etapa de ejecución, donde las actividades de EaD se llevan adelante siguiendo la estrategia trazada, con los ajustes que sean necesarios para alcanzar los objetivos educacionales.

\section{Discusiones}

La aplicación del modelo propuesto en este artículo toma en consideración que la mayoría de las plataformas de software para $\mathrm{EaD}$, claramente las que se basan en software libre, cuentan con ambientes para foros de discusión y comunidades, según Araujo, Cavalcanti y Anjos (2006), lo que permite la creación de comunidades virtuales entre los participantes de EaD. Como estos dos ambientes presentan características 
bastante similares, se puede inferir que tanto las barreras como los factores de incentivo a la participación que han sido identificados para las $\mathrm{CoP}$ son relevantes para la EaD.

Hay que poner de relieve que, al observar las barreras listadas en las Tablas 1, 2, 3 y 4, se percibe fácilmente que no todas guardan relación con las metas de la EaD pues son específicas de las comunidades de práctica. En este caso, sólo las barreras que corresponden a la $\mathrm{EaD}$ deben ser consideradas, quedando de lado las que están ligadas sólo a las CoP.

En la revisión de literatura fueron identificados treinta (30) factores de incentivo a la participación en las comunidades virtuales. Esos factores fueron presentados sin ninguna tentativa de categorización, aunque sería posible agruparlos con fines de simplificación y de análisis.

Así, en la etapa de desarrollo de la estrategia de participación se debe ponderar qué factores de incentivo se aplican específicamente al caso estudiado para considerar los que son relevantes y dejar de lado los que no corresponden.

\section{Conclusión}

Creemos que el modelo propuesto en el presente artículo, aunque aún no haya sido puesto a prueba en una aplicación real, puede redundar en ganancia de efectividad en las iniciativas de la $\mathrm{EaD}$, ya que se trata de una trasposición de algunas prácticas que vienen siendo adoptadas en una estructura que presenta gran similitud con la $\mathrm{EaD}$ : la de las comunidades de práctica.

Los supuestos asumidos en este estudio nos llevan a pensar que, si el modelo se adopta en un ambiente de EaD donde la creación de comunidades virtuales sea un factor de alto impacto en el resultado de aprendizaje, la efectividad del proceso con seguridad aumentará, lo que contribuirá a la reducción de los índices de deserción.

En trabajos futuros se podrá tratar el agrupamiento de los factores de incentivo a la participación y estudiar de forma más detallada su adecuación a las actividades de $\mathrm{EaD}$, por medio de la aplicación en un caso real.

Para ello debe ser investigarse el comportamiento social y cultural del grupo específico de alumnos, verificando su grado de escolaridad, franja de edad y motivos que los llevaron a elegir el curso. El estudio del público destinatario facilita la identificación de las barreras y la creación de incentivos a la participación, que significarán, como recompensas, el reconocimiento, el comprometimiento y el aumento de la autoestima y la autoeficacia del alumno, contribuyendo positivamente a su aprendizaje.

\section{Referencias}

Almeida, M. E. B. (2003) "Educação a distância na internet: abordagens e contribuições dos ambientes digitais de aprendizagem". São Paulo, Revista Educação e Pesquisa, v. 29 , n. 2, pp. 327-340.

Araujo, A. M., Cavalcanti, A. C., Anjos, L. (2006) "Um Estudo Comparativo sobre Plataformas de EaD Baseadas em Software Livre", Simpósio Brasileiro de Informática na Educação, Anais do SBIE, pp. 1-3. 
Ardichvili, A., Page, V., Wentling, T. (2003) "Motivation and Barriers to Participation in Virtual Knowledge-Sharing Communities of Practice", Journal of Knowledge Management, 7(1), pp. 64-77.

Ardichvili, A. (2008) "Learning and Knowledge Sharing in Virtual Communities of Practice: Motivators, Barriers and Enablers", Advances in Developing Human Resources, v. 10, n. 4, pp. 541-554.

Beenen G., Ling K.,Wang, X., Chang, K., Frankowski, D., Resnick, P., Kraut, R. (2004) "Using Social Psychology to Motivate Online Contributions", The 2004 ACM Conference on Computer Supported Cooperative Work, CSCW 2004, Chicago, Illinois, pp. 1-10.

Chan, C. M. L., Bhandar, M., Oh, L. B., Chan, H. C. (2004) "Recognition and Participation in a Virtual Community", Proceedings of the 37th Hawaii International Conference on System Sciences, Kona, Hawaii, pp. 1-10.

Detoni, D., Araujo, R. M., Cechinel, C. (2014). Predição de Reprovação de Alunos de Educação a Distância Utilizando Contagem de Interações. XXV Simpósio Brasileiro de Informática na Educação.

Favero, R. V. M., Franco, S. R. K. (2006) "Um estudo sobre a permanência e a evasão na Educação a Distância”, Novas Tecnologias em Educação, v. 4, n. 2, pp. 1-10.

Gannon-Leary, P., Fontainha, E. (2007) "Communities of Practice and Virtual Learning Communities: benefits, barriers and success factors”, eLearning Papers, n. 5, pp. 114.

Goman, C. K. (2002) “Five Reasons People Don’t Tell What They Know”, Knowledge Management Magazine.

Hara, N. (209) "Communities of Practice: Fostering Peer-to-Peer Learning and Informal Knowledge Sharing in the Work Place". Berlin: Springer-Verlag.

Heidrich, L., Barbosa, J., Rigo, S., Cambruzzi, W., Ribeiro, G. (2014). Diagnóstico do Comportamento dos Aprendizes na Educação a Distância com Base no Estilo de Aprendizagem. XXV Simpósio Brasileiro de Informática na Educação.

Hoadley, C. M., Pea, R. D. (2001) "Finding the Ties that Bind: Tools in Support of a Knowledge-Building Community", in Building Virtual Communities: Learning and Change in Cyberspace, New York: Cambridge University Press.

INEP (2013) Instituto Nacional de Estudos e Pesquisas Educacionais Anísio Teixeira, “Censo da Educação Superior”, Brasília: INEP, 2013.

Kimble, C., Li, F., Barlow, A. (2000) "Effective Virtual Teams Through Communities of Practice", Management Science Research Paper, n. 9, Strathclyde Business School, pp. 1-15.

Mahar, G. J. (2007) "Factors Affecting Participation in Online Communities of Practice", PhD Thesis, University of Waterloo, Canada.

Manhães, L. M. M., Cruz, S. M. S., Costa, R. J. M., Zaveleta, J., and Zimbrao, G. (2011). Previsão de Estudantes com Risco de Evasão Utilizando Técnicas de Mineração de Dados. Anais do XXII SBIE. 
McKenzie-Mohr, D., Smith, W. (1999) "Fostering Sustainable Behavior: An Introduction to Community-Based Social Marketing", New Society Publishers, Gabriola Island, Canada.

Moore, M. G. (2007) “Handbook of Distance Education”, Mahwah: Lawrence Erlbaum Associates.

Moran, J. M. (2007) “A educação que desejamos: novos desafios e como chegar lá”. São Paulo: Papirus.

Nonnecke, B., Preece, J., Andrews, D., Voutour, R. (2004) “Online Lurkers Tell Why”, Proceedings of the Tenth Americas Conference on Information Systems, New York, pp. 1-7.

Palloff, R. M., Pratt, K. (2007) "Building online learning communities: Effective Strategies for the Virtual Classroom”. San Francisco: Jossey-Bass.

Peters, O., Keegan, D. (1994) "Otto Peters on distance education: the industrialization of teaching and learning”. London: Rutledge Taylor \& Francis.

Ramos, J. L. C., Rodrigues, R. L., Silva, J. C. S., Gomes , A. S. (2014). Analisando Fatores que Afetam o Desempenho de Estudantes Iniciantes em um Curso a Distância. XXV Simpósio Brasileiro de Informática na Educação.

Teigland, R., Wasko, M. M. (2004) "Extending Richness with Reach: Participation and Knowledge Exchange in Electronic Networks of Practice", in Knowledge Networks: Innovation through Communities of Practice, Idea Group Inc.

Wasko, M. M., Faraj, S. (2000) “'It Is What One Does': Why People Participate and Help Others in Electronic Communities of Practice", Journal of Strategic Information System, v. 9, pp. 155-173.

Wenger, E., McDermott, R., Snyder, W. (2002) "Cultivating Communities of Practice: a Guide to Managing Knowledge”. Boston: Harvard Business School Press.

Wenger, E., (2010) "Communities of Practice: A Brief Introduction", http://www.ewenger.com/theory/index.htm, June. 